\title{
Advanced Wireless Sensor Nodes - MSFC
}

Kosta Varnavas / Jeff Richeson

Marshall Space Flight Center / Jacobs

Electronic Design Branch

Mail Stop ES36

MSFC,Al 35812

Ph: 256-544-2638 , 256-961-0128

Email: kosta.varnavas@nasa.gov, james.j.richeson@nasa.gov

\begin{abstract}
NASA field center Marshall Space Flight Center (Huntsville, AL), has invested in advanced wireless sensor technology development. Developments for a wireless microcontroller back-end were primarily focused on the commercial Synapse Wireless family of devices. These devices have many useful features for NASA applications, good characteristics and the ability to be programmed Over-The-Air (OTA). The effort has focused on two widely used sensor types, mechanical strain gauges and thermal sensors. Mechanical strain gauges are used extensively in NASA structural testing and even on vehicle instrumentation systems. Additionally, thermal monitoring with many types of sensors is extensively used. These thermal sensors include thermocouples of all types, resistive temperature devices (RTDs), diodes and other thermal sensor types. The wireless thermal board will accommodate all of these types of sensor inputs to an analog front end. The analog front end on each of the sensors interfaces to the Synapse wireless microcontroller, based on the Atmel Atmega128 device. Once the analog sensor output data is digitized by the onboard analog to digital converter $(\mathrm{A} / \mathrm{D})$, the data is available for analysis, computation or transmission. Various hardware features allow custom embedded software to manage battery power to enhance battery life. This technology development fits nicely into using numerous additional sensor front ends, including some of the low-cost printed circuit board capacitive moisture content sensors currently being developed at Auburn University.
\end{abstract}

Key words

Sensors, wireless, strain gauge, thermocouple 


\section{Introduction}

Internal Research and Development (IRAD) money has supported the development of wireless sensors at Marshall Space Flight Center (MSFC). First use of the technology prototypes was to demo the sensors on ground test articles in the structures lab and materials lab. The goals were to verify accuracy, usability and reliability from an RF data throughput stance.

After looking at what the industry had to offer, the synapse wireless SM200 integrated Zigbee protocol RF microcontroller was chosen. It was chosen based on its capabilities to be programmed in python using a built in application programmers interface, its relatively low power for receive and transmit, as well as its ability to upload new applications over the air (OTA).

This resulted in the first generation of a strain gauge and a $\mathrm{J}, \mathrm{K}$ thermocouple sensor boards. The first generation strain gauges had:

- 6 gauges all powered at same time.

- $\quad$ Strain gauged excitation voltage was straight off main battery rail.

- $\quad$ Op amp only has a 200 gain. This is a fixed gain set by on board resistor

- No shunt or other method for onboard calibration

- $\quad$ No Power Management.

These worked very well on a variety of test platforms as directly compared to known good reference sensors. The next generation was built to reduce size, and manage battery power better than the first generation.

\section{Second Generation Sensors}

The sensors are based on the synapse wireless RF microcontrollers with Zigbee protocol stack and python API.

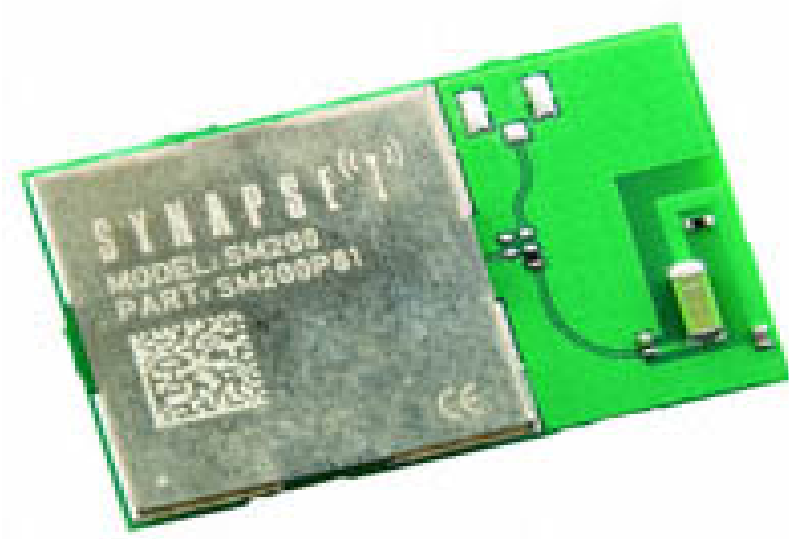

This is approximately 1.25 inches by 0.75 inches. To reduce overall size of the boards and power the second generation strain gauge has:

- $\quad$ Only 1 gauge input.

- Has an independent constant voltage regulator driving the excitation voltage.

- $\quad$ Power Management Hardware

- Op Amp has much larger and adjustable gains.

- $\quad$ Power management software.

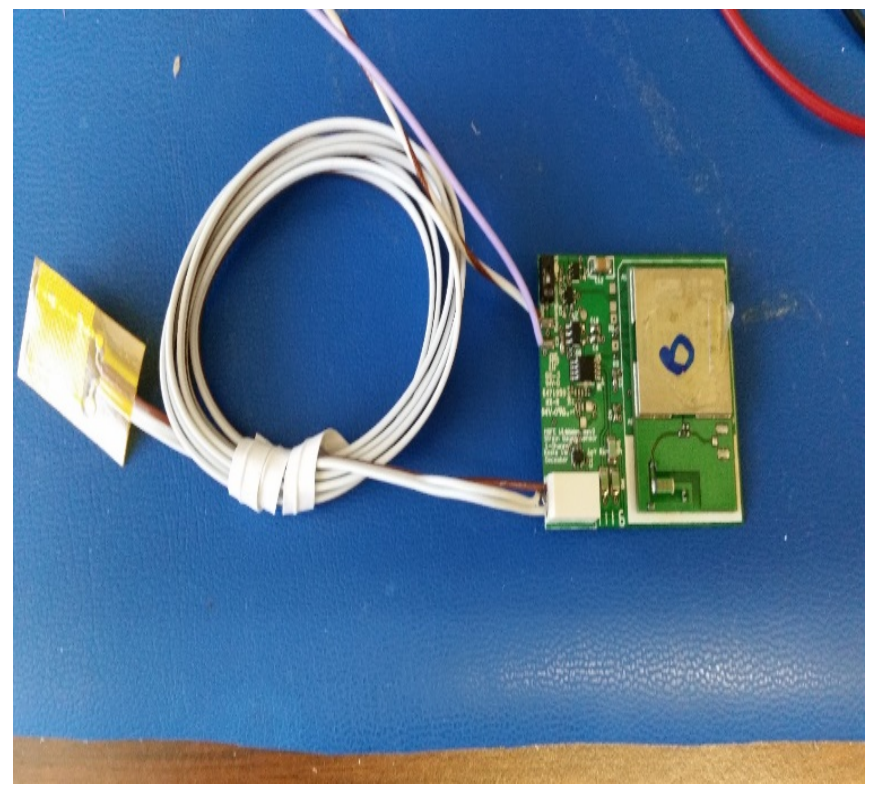


The second generation thermal sensor uses a chip that can measure:

- virtually all standard (type $\mathrm{B}$, E, J, K, N, S, R, T) or custom thermocouples.

- Automatically compensate for cold junction temperatures and linearize the results.

- 2-, 3-, or 4-wire RTDs.

- Thermistors

- Diodes

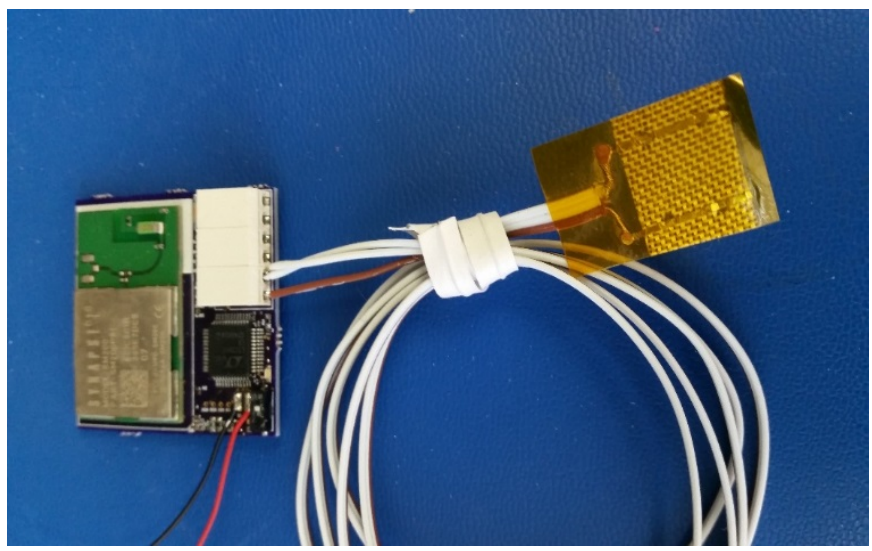

In addition to the sensors themselves, a battery charging station was designed and built to allow multiple sensors to be placed into the charger to recharge the lithium ion batteries. The sensors, pictured, along with the battery that is sized to the sensor board size, were all placed into a 3D printed box for field testing.

\section{A. Composite Shell Field Test}

Field testing for the thermal sensors is ongoing. The field testing for the strain gauges met with some technical difficulty. The first generation sensor field testing went very well. The second generation field test went well too, when using one wireless sensor at a time. The data always compared very well with the reference sensors. Lab testing of up to 25 sensors was pretty successful also. The lab testing was not testing the sensor data as much as it was testing how 25 nodes on the network worked. In the lab environment all worked well. But the field test with 20 proved more troublesome.

Structures lab had a large composite material shell that they were going to stress to the buckling point. They allowed our wireless strain gauges to be mounted near their normal gauges. The plan was to get data for as long as the batteries lasted and be able to do a direct compare between the wireless sensors and the reference.
Unfortunately, the sensors never lasted but a few minutes as they began to drop out. The RF interference was too great. It is believed that the RF environment in that lab is significantly nosier than our development lab.

However, this was not a loss. One of the big questions to answer is how to handle a noisy RF environment for many sensor nodes. The ultimate goal is to be able to have hundreds on the mesh network at one time. Also, the test was done in the harshest manner. All nodes were allowed to talk as they got data, in a live stream scenario with minimal collision avoidance. This is important information.

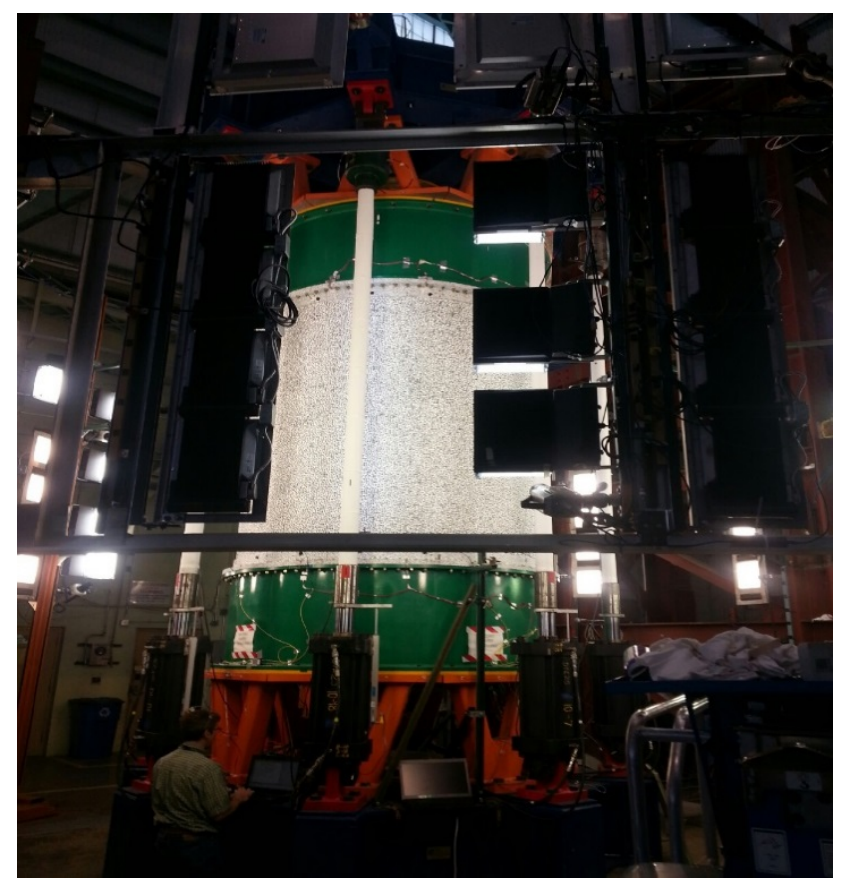

\section{B. Test Lab Pull Test}

The pull test had been done before with the first generation sensors and the data matched well between the wireless node and the reference. However, a similar test, with only 5 nodes in the same noisy environment as the composite test resulted in similar problems. As four of the nodes dropped out the fifth worked very well! 


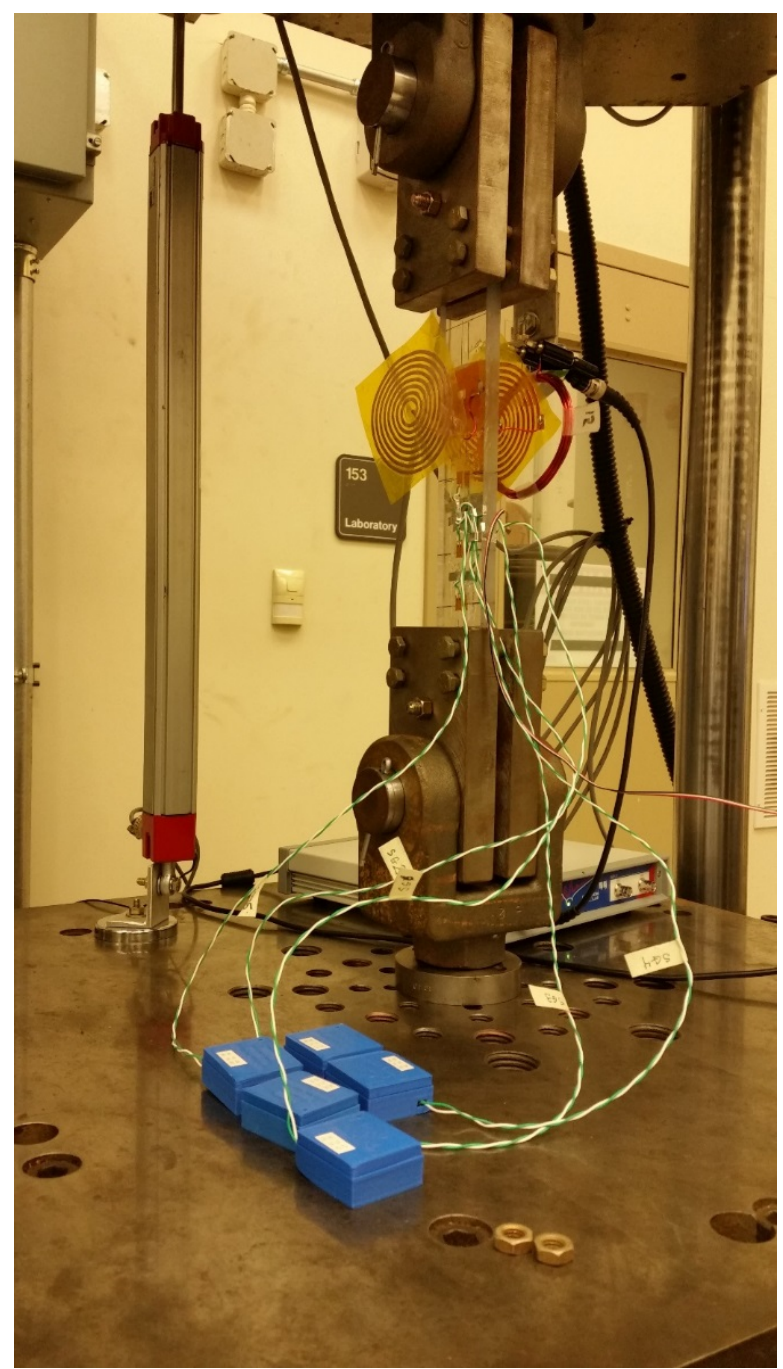

\section{Next Steps}

The next steps are quite clear. Advanced anti-collision techniques will have to be developed. This is a big part of the technology development for large networks of wireless sensors. One of the best techniques that will be implemented in the 2017 time frame is to implement a polling method to get the data. That way only one node at a time is transmitting and only when told to do so.

However, there is still some interest in experimenting with anti-collision software enhancements, along with utilizing the ability of each node to "sniff" the RF environment and change channels to one with the least amount of RF interference. There are a variety of techniques and combinations of techniques that will be explored.

These methods will be utilized on all types of sensor nodes as the wireless mesh network will behave the same regardless of the data being sensed.
Additionally, a space act agreement has been signed with Auburn University to explore the integration of these wireless mesh nodes and control software, with Auburn developed senor devices. Specifically these sensors are unique moisture sensors that can be applied to many applications in the agriculture and environmental sciences.

\section{Conclusion}

In spite of some failures to get good data with a large number of wireless nodes, the underlying hardware technology is working well. The data taken per node individually is as good as the reference sensors and with the second generation, good power management and gain control has been introduced and demonstrated. More work has to be done to have a large number of nodes on a network at the same time reliably enough to get the data through and last for a reasonable period of time. 